\title{
Quantum Mechanical Simulation of Charge Transport in Very Small Semiconductor Structures
}

\author{
M. C. YALABIK, G. NEOFOTISTOS, KARIM DIFF, HONG GUO, AND \\ JAMES D. GUNTON, MEMBER, IEEE
}

\begin{abstract}
A quantum mechanical simulation method of charge transport in very small semiconductor devices, based on the numerical solution of the time-dependent Schrödinger equation (coupled self-consistently to the Poisson equation to determine the electrostatic potential in the device), is presented. Carrier transport is considered within the effective mass approximation, while the effects of the electron-phonon interaction are included in an approximation that is consistent with the results of the perturbation theory and gives the correct two-point time correlation function. Numerical results for the transient behavior of a planar ultrasubmicrometer three-dimensional GaAs MESFET (gate length of $26 \mathrm{~nm}$ ) are also presented. They indicate that extremely fast gate-step response times (switching times) characterize such shortchannel GaAs devices.
\end{abstract}

\section{INTRODUCTION}

QIMULATION of charge transport in very small geoDmetries has important applications in the field of semiconductor devices, where the prediction and analysis of device operations can be carried out more economically through the use of computers. Several techniques have been used for the simulations, depending on the way the physics of the charge transport is modeled. If the mean free path of the carriers is much smaller than the typical geometric features of the structure, the diffusion equation may be used to describe the dynamics of the charges under the influence of a self-consistently determined electrostatic potential. When the mean free path becomes comparable to the device size, the semiclassical Boltzmann equation may be solved approximately either through the use of equations for its moments [1] or through a Monte Carlo simulation [2]. In both methods, it is assumed that the quantum mechanical wavelength of the carriers is small compared to the size of the structure. However, the current trend toward miniaturization in the microelectronics technology, combined with the developments in the processing of new electronic materials with lower car-

Manuscript received August 17, 1988. This work was supported by the U.S. Office of Naval Research under Grant N00014-83-K-0382 and by an allocation of computer time by the Pittsburgh Supercomputer Center. The review of this paper was arranged by Associate Editor S.-S. Pei.

M. C. Yalabik is with the Department of Physics, Bilkent University, Ankara, Turkey

G. Neofotistos and K. Diff are with the Physics Department and the Center for Advanced Computational Science, Temple University, Philadelphia, PA 19122.

H. Guo and J. D. Gunton are with the Department of Physics, Lehigh University, Bethlehem, PA 18105

IEEE Log Number 8826320. rier effective masses (which results in high mobilities and long quantum mechanical wavelengths), indicate that many electronic devices will soon be operating in a regime different from that which can be simulated by the semiclassical Boltzmann transport equation. A number of laboratories have already reported devices with sizes comparable to the quantum mechanical wavelengths of the carriers.

At present, there is a very limited number of tools available for the numerical simulation of quantum mechanical charge transport in a given geometry. For one-dimensional problems, the Wigner function [3] can be utilized to follow the electron distribution function [4]. This method has the advantage that the incoming and outgoing carriers are separate in the analysis, which results in relatively simple boundary conditions. A second advantage is that the dissipation effects due to inelastic scattering of the carriers can be included in an "intuitive" way, since the equation of motion has a striking similarity to the semiclassical Boltzmann equation. A disadvantage of the approach is its somewhat complicated stability properties. The Wigner function approach has at best a limited applicability to higher dimensions, mainly due to the present limitations of computer equipment (a $2 \times d$-dimensional mesh is necessary for a $d$-dimensional problem). One can construct equations for the moments of the Wigner function [5] and solve these equations for a given geometry. This is a truncation scheme and has not found wide use. Recently, there has been some success in numerically implementing path integrals for quantum mechanical transport. The main advantage of this method is the accurate handling of some types of dissipative effects. However, the amount of computational time needed is prohibitively large (even for simple cases).

Availability of supercomputer resources now makes it feasible to carry out a simulation of charge transport in a given geometry through the solution of the Schrödinger equation. The major disadvantages of this approach are the numerical problems encountered in specifying the boundary conditions at the contacts of the device, and the fundamental problems associated with the incorporation of dissipative effects into the Schrödinger equation. Although more work is necessary to understand these difficulties, certain approximations are available for computational purposes. 
In this work, we describe such a simulation, in which we numerically integrate the time-dependent Schrödinger equation for carriers in a GaAs MESFET. Electrons in the $\Gamma$ and $L$ valleys are considered within the effective mass approximation. The carriers move under the influence of the electrostatic potential determined self-consistently through the simultaneous solution of the Poisson equation. The carrier density is assumed to be sufficiently low so that interactions among the electrons (apart from the self-consistent electrostatic potential) can be neglected, and hence the system can be described by a superposition of single particle states. The effects of the electronphonon interaction are included in an approximation discussed below, which is consistent with the results of perturbation theory and yields the correct two-point function in equilibrium. Intravalley optical and acoustical as well as equivalent and non-equivalent intervalley scattering events are considered in the simulation. We describe the method used and the approximations involved in the following sections. Some initial results of the simulation are also reported.

\section{Numerical Method and Model Used}

We solve the time-dependent Schrödinger equation

$$
i \hbar \frac{\partial \psi_{i}}{\partial t}=H_{i} \psi_{i}+V_{i j}^{e-p h} \psi_{j}
$$

where $\psi_{i}\left(\psi_{j}\right)$ is the wave function (the indices $i, j$ label the conduction band valleys, $\Gamma$ or $L$, that the electron is in), $h$ is Planck's constant, $t$ is the time, $V_{i j}^{e-p h}$ is the potential corresponding to the intervalley electron-phonon scattering, and $H_{i}$ is the Hamiltonian in the $i$ th valley

$$
H_{i}=-\frac{\hbar^{2}}{2 m_{i}^{*}} \nabla^{2}-e \cdot \Phi+V_{i}^{e-p h} .
$$

Here, $m_{i}^{*}$ is the electron effective mass in the $i$ th valley, $\Phi$ is the electrostatic potential, and $V_{i}^{e-p h}$ is the potential corresponding to the electron-phonon scattering in this valley. The electrostatic potential is determined by solving the Poisson equation (subject to the boundary conditions of the device) with a source term that contains the charge distribution at that instant

$$
\epsilon \nabla^{2} \Phi=e\left(\psi_{\Gamma}^{*} \psi_{\Gamma}+\psi_{L}^{*} \psi_{L}\right)+n
$$

In this equation, $\epsilon$ is the dielectric permittivity of GaAs, and $n$ represents the fixed background dopant charge distribution. Note that the wave functions are normalized so that $\psi(x)^{*} \psi(x)$ represents the total density of particles at point $x$ in the corresponding valley.

A correct treatment of the last term in (2) is a difficult fundamental problem. Before going into the details of our approximation of this term, we will discuss briefly the numerical integration method. If there is no electronphonon coupling term $\left(V_{i}^{e-p h}=0\right)$, the time development of the wave function can be obtained by

$$
\psi(t+\Delta t) \approx e^{-(i \Delta t / h)(K-e \cdot \Phi)} \psi(t)
$$

or

$$
\begin{aligned}
\psi(t+\Delta t) \approx & e^{(-(i \Delta t / 2 \hbar) K)} e^{(i \Delta t / h) e \cdot \Phi} \\
& \cdot e^{(-(i \Delta t / 2 \hbar) e \cdot \Phi, K)} \psi(t)
\end{aligned}
$$

where $K$ stands for the kinetic energy term in (2). Note that the decomposition shown in (4b) preserves the time reversal symmetry of the Schrödinger equation. Since the term associated with the kinetic energy is very simple in momentum space, that part of the operation was carried out in momentum space. This procedure enables one to include effects such as non-parabolicities of the band structure in the simulation, although no such attempt has been made in the present study. The terms in (4b) associated with the electrostatic potential can best be handled in position space, so that our integration procedure involves two Fourier transform operations per time step (corresponding to going back and forth between position and momentum spaces).

Concerning the electron-phonon coupling, the determination of the dissipative effects of the phonons on the electron states is a difficult fundamental problem in quantum transport theory [6]-[9]. Although there has been considerable formal progress, it is computationally difficult to implement these formalisms (such as the path integral approach) in numerical simulations for devices. As a consequence, we consider here a simple approximation scheme for the electron-phonon interaction that allows us to simulate such dissipative effects within the context of a Schrödinger equation approach. The approximation consists of two parts. The first part involves the modification of the occupation numbers of the electron states (in momentum space) at each step, consistent with the electron-phonon scattering rates one obtains from perturbation theory, i.e.,

$$
\begin{aligned}
\frac{\partial\left|\alpha_{k}\right|^{2}}{\partial t} \approx & \frac{2 \pi}{\hbar} \sum_{k^{\prime}}\left(\left|V_{k k^{\prime}}\right|^{2}\left|\alpha_{k^{\prime}}\right|^{2}-\left|V_{k^{\prime} k}\right|^{2}\left|\alpha_{k}\right|^{2}\right) \\
& \times \delta\left(E(k)-E\left(k^{\prime}\right) \pm \hbar \omega\right)
\end{aligned}
$$

where $\alpha_{k}$ is the amplitude of the $k$ component of the wave function, $V_{k k^{\prime}}$ is the matrix element of the electron scattering from state $k^{\prime}$ to $k$ due to phonons, and the $\delta$ function represents the energy conservation due to a phonon (of energy $\hbar \omega$ ) absorption or emission process. It should be noted that the computation of these rates involves a long time limit (and hence conservation of energy) that obviously should not hold for the small time steps of this simulation. It could also be argued that the approximation would make better sense if transitions between the eigenstates of the Hamiltonian at that particular time (which unfortunately are not readily available) were considered instead of transitions between the momentum states. Nevertheless, the approximation we have used guarantees a correct equilibrium distribution and considerably stabilizes the numerical procedure.

Our second approximation is to assume that the effect of the phonons on the phase of the electrons is described 
by a random noise term

$$
\phi_{k} \approx \xi_{k}(t)
$$

where $\phi_{k}$ is the phase of the $k$ component of the wave function, and $\xi_{k}$ represents the noise term. In practice, this is implemented by the following procedure: at each time step, the phase is modified by the addition of a term that is randomly distributed between $-\Gamma_{k}$ and $\Gamma_{k}$. Identification of $\Gamma_{k}$ will be made below, using the equilibrium two-point time correlation function that one obtains as a result of this approximation. With this approximation, the phase of the $k$ component of the wave function is expected to have a diffusive behavior and will be distributed as

$$
\Phi_{k}\left(\phi_{k}\right)=A e^{\left(-\left(\phi_{k}^{2} / 2 \Gamma_{k} t\right)\right)}
$$

where $\Phi$ represents the probability density function for the distribution of $\phi_{k}$ at time $t$, relative to its initial value at $t$ $=0$, and $A$ is a normalization constant. One can then compute the expectation value of the equilibrium twopoint function for the homogeneous system in the presence of the electron-phonon interaction

$$
\begin{aligned}
G(k, R, \omega, t)= & \int d \tau \int d Q\left\langle\left\langle\alpha_{k+(Q / 2)(\mathrm{eq})}^{*}\left(t+\frac{\tau}{2}\right)\right.\right. \\
& \left.\left.\cdot \alpha_{k-(Q / 2)(\mathrm{eq})}\left(t-\frac{\tau}{2}\right) e^{i(\omega \tau+Q R)}\right\rangle\right\rangle
\end{aligned}
$$

where $\langle\langle\cdots\rangle\rangle$ denotes statistical averaging over the phases of the wave-function components. This quantity will not have any position $(R)$ or time dependence, due to the translational invariance in space and time. The detailed balance condition corresponding to the transition rates in (5) implies that, at equilibrium, the magnitude of the wave function is given by

$$
\left|\alpha_{k_{\mathrm{eq}}}\right|=C e^{-\left(E(k) / 2 k_{B} T\right)}
$$

where $C$ is a normalization constant, $k_{B}$ is the Boltzmann constant, and $T$ is the temperature (due to the assumption of low density noninteracting particles). From

$$
\alpha_{k}(t)=C e^{-\left(E(k) / 2 k_{B} T\right)+i \phi_{k}(t)}
$$

and noting that

$$
\begin{aligned}
\left\langle\left\langle e^{i\left(\phi_{\mathrm{k}}(t)-\phi_{\mathrm{k}^{\prime}}\left(t^{\prime}\right)\right)}\right\rangle\right\rangle & =\delta\left(k-k^{\prime}\right) \int d \phi A e^{\mathrm{i} \phi-\left(\phi^{2} / 2 \Gamma_{k} t\right)} \\
& =\delta\left(k-k^{\prime}\right) e^{-\Gamma_{k}\left|t-t^{\prime}\right|}
\end{aligned}
$$

one finally obtains for the two-point function

$$
G(k, \omega)=\frac{e^{-E(k) / k_{B} T}}{\omega-E(k)-i \Gamma_{k}} .
$$

Comparison of this expression with the field-theoretical result [8] leads to the identification of $\Gamma_{k}$ as the imaginary part of the self-energy due to electron-phonon scattering, apart from the correction of the quasi-particle energy due to the real part of the self-energy (which we may assume is already included in $E(k))$. This procedure therefore results in an equilibrium distribution function for the homogeneous system that is consistent with the field-theoretical result. At equilibrium, $\Gamma_{k}$ is given by

$$
\Gamma_{k_{\text {equil. }}}=\sum_{k^{\prime}}\left|V_{k k^{\prime}}\right|^{2} \delta\left(E(k)-E\left(k^{\prime}\right) \pm \hbar \omega\right) n_{\text {equil }}\left(k^{\prime}\right)
$$

where $n_{\text {equil }}\left(k^{\prime}\right)$ is the equilibrium electron distribution function.

Away from equilibrium, we have used for the imaginary part of the unknown non-equilibrium self-energy the approximation

$\Gamma_{k} \approx \sum_{k^{\prime}}\left|V_{k k^{\prime}}\right|^{2} \delta\left(E(k)-E\left(k^{\prime}\right) \pm \hbar \omega\right)\left|\alpha_{k^{\prime}}(t)\right|^{2}$.

Our treatment of the electron-phonon interaction would be expected to give good results for systems weakly coupled to the phonon field, and not too far from equilibrium. Since our theory is phenomenological, it is difficult to assess the degree of its validity more generally.

\section{Results}

A planar GaAs MESFET has been simulated using the method described in the previous section. The gate length of the device was chosen to be $26 \mathrm{~nm}$. GaAs MESFET's, grown by $\mathrm{MBE}$ on semi-insulating substrates, with gate lengths as short as $27.5 \pm 2.5 \mathrm{~nm}$ have currently been fabricated [10]. The lengths of the source and drain were considered to be both $24 \mathrm{~nm}$. The complete list of device parameters used in our simulation is given in Table I. Physical parameters such as effective masses, valley separation, phonon energies, etc., are given in [11].

The doping concentration of the $\mathrm{n}^{+}$contact regions is taken to be nonuniform (Gaussian-like) with a peak concentration of $1 \times 10^{17} \mathrm{~cm}^{-3}$. The incorporation of such a profile (representative of ion-implanted devices, although unrealistically implemented in our simulation, with respect to its actual spread), smoothes out the problems (i.e., spikes in the particle distribution) encountered at the boundaries of the much steeper, wall-like, doping profiles used in previous simulations [12]. In addition, a higher, more realistic doping has not been considered since it necessitates the use of the Pauli exclusion principle to take into account the degeneracy effect. The doping concentration of the conduction channel was taken to be uniform with a value of $2 \times 10^{16} \mathrm{~cm}^{-3}$. The overall doping profile considered in this simulation is shown in Fig. 1.

During the initial time steps of the device operation (gate is "on"; see Fig. 2), thermally distributed electrons are injected at the source contact into the $n^{+}$region of the device. The outgoing components of the wavefunction are identified and removed at these contacts. However, the use of this kind of boundary conditions has been shown to lead to the development of "spikes" of the particle distribution at the contacts [12]. In order to overcome this problem, we have kept the wave-function constant at these 
TABLE I

Numerical and Device Parameters for a Three-Dimensional TimeDependent Simulation of a Planar GaAs MESFET

\begin{tabular}{lccc}
\hline \hline Device dimensions: & 127 & $\mathrm{~nm}$ \\
along the -x (source-drain) axis & 63 & $\mathrm{~nm}$ \\
along the -y (contacts-substrate) axis & 16 & $\mathrm{~nm}$ \\
along the -z (width of the device) axis & 26 & $\mathrm{~nm}$ \\
\hline Gate length & 24 & $\mathrm{~nm}$ \\
Source length & 24 & $\mathrm{~nm}$ \\
Drain length & $2 \times 10^{16}$ & $\mathrm{~cm}^{-3}$ \\
Channel doping concentration & $1 \times 10^{17}$ & $\mathrm{~cm}^{-3}$ \\
Peak concentration at $\mathrm{n}^{+}$regions & 300 & ${ }^{\circ} \mathrm{K}$ \\
Temperature & $63 \times 127 \times 16$ & \\
\hline Mesh size & 1 & $\mathrm{~nm}$ \\
Mesh spacings & $4 \times 10^{-16}$ & $\mathrm{~s}$ \\
Time step & & \\
\hline
\end{tabular}

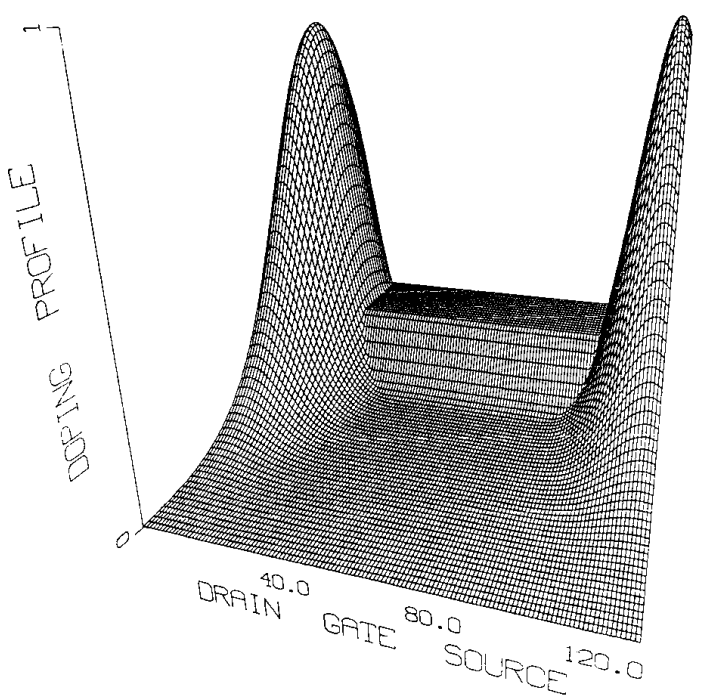

Fig. 1. Doping profile (normalized) used in our simulation. The dopant concentration at the peaks of the $n^{+}$regions is taken to be $1 \times 10^{17} \mathrm{~cm}^{-3}$ The concentration in the conductive channel is $2 \times 10^{16} \mathrm{~cm}^{-3}$. In this graph, the device is viewed from the substrate region.

contacts after the first time steps. This is an ad hoc, but reasonable, constraint.

The simulation was carried out on a $63 \times 127 \times 16$ mesh (with 1-nm mesh spacing), with periodic boundary conditions in the $-z$ direction (which represents the width of the device; see Table I), where translational invariance is expected. This choice of the mesh spacing satisfies the sampling requirements [13] of the numerical method used, and minimizes the numerical error due to the discretization of the kinetic energy operator [14]. A time step of 4 $\times 10^{-16} \mathrm{~s}$ has been used. With this time step, a simulation

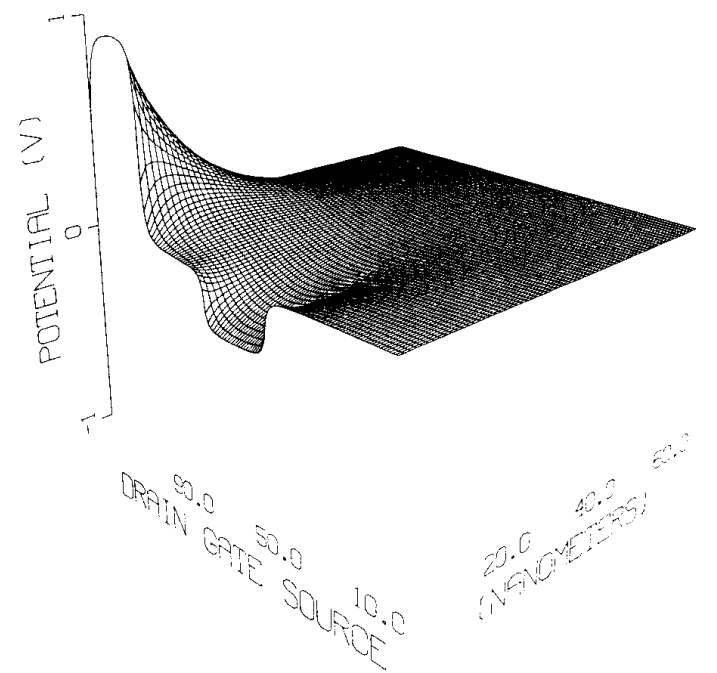

Fig. 2. Potential energy profile (as viewed from the contacts region) after the device has been switched on. The values of the potentials are $V_{\text {source }}$ $=0 \mathrm{~V}, V_{\text {gate }}=-0.3 \mathrm{~V}, V_{\text {drain }}=1 \mathrm{~V}$

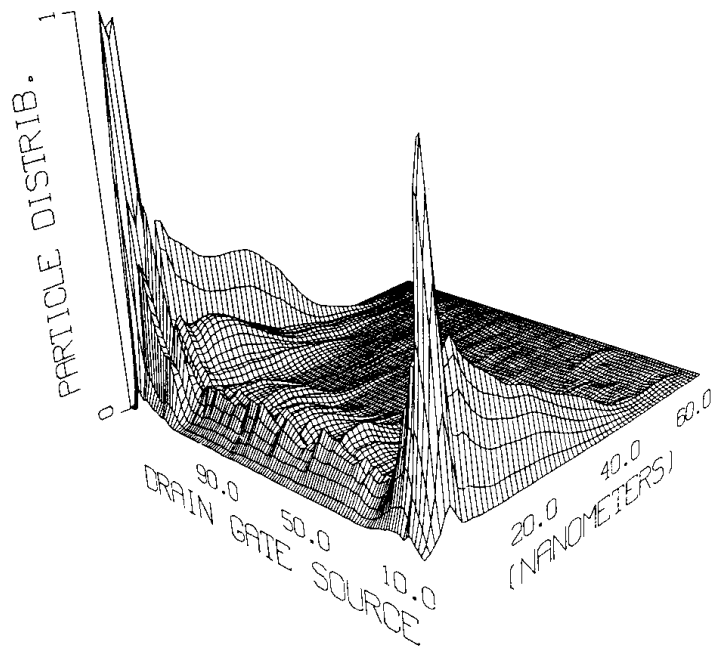

Fig. 3. Carrier density distribution in the device at time $t=4 \mathrm{fs}$ (just before we switch the device off). Carriers in both $\Gamma$ and $L$ valleys have been taken into account $\left(|\psi(x)|^{2}=|\psi(x)|_{\Gamma}^{2}+|\psi(x)|_{L}^{2}\right)$. The external bias is that of Fig. 2.

corresponding to $1 \mathrm{ps}$ takes approximately $70 \mathrm{~h}$ of computer time (system units) on a CRAY X-MP (at present, 90 percent of the computer time is spent in the handling of the electron-phonon interaction; this amount will be considerably reduced in the future as the code is further optimized). We have, so far, performed runs out to 0.01 ps. Fig. 3 shows the carrier density distribution obtained at a time $t=4 \mathrm{fs}$ after the device has been switched on ( $V_{\text {gate }}=-0.3 \mathrm{~V}, V_{\text {drain }}=1 \mathrm{~V}, V_{\text {source }}=0 \mathrm{~V}$; see Fig. $2)$. After this time, the gate is instantaneously switched ("off") to $V_{\text {gate }}=0.1 \mathrm{~V}$ (with $V_{\text {drain }}=1 \mathrm{~V}$ and $V_{\text {source }}=$ $-0.3 \mathrm{~V}$; see Fig. 4). Fig. 5 shows the carrier density 


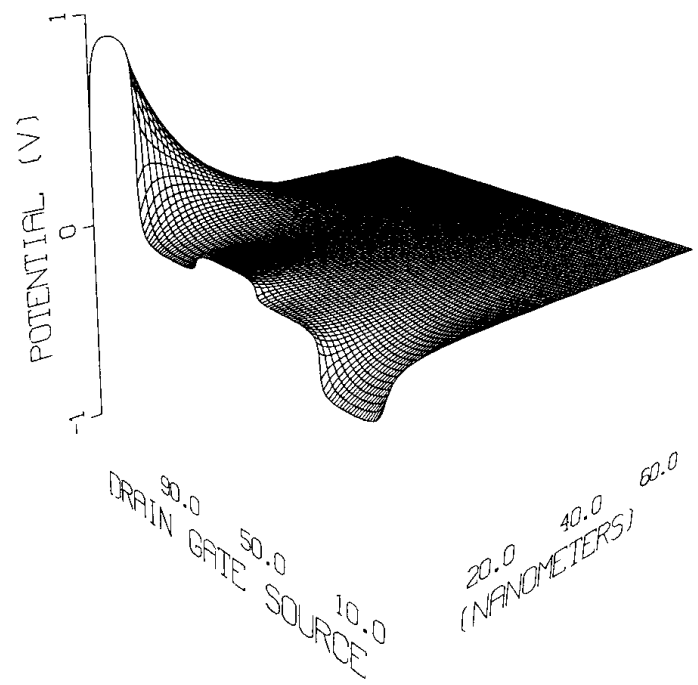

Fig. 4. Potential energy profile after the device has been switched off. The values of the potentials are $V_{\text {source }}=-0.3 \mathrm{~V}, V_{\text {gate }}=0.1 \mathrm{~V}, V_{\text {drain }}=1$ V.

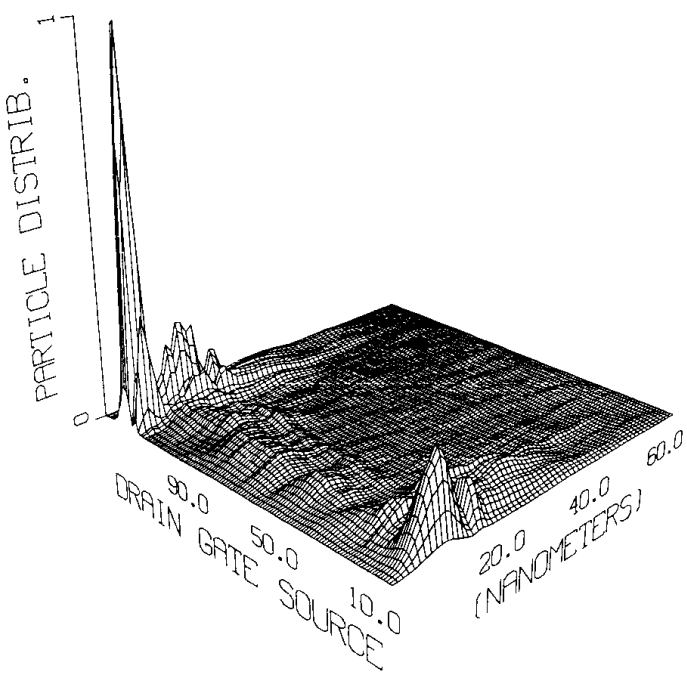

Fig. 5. Carrier density distribution at time $t=6.4 \mathrm{fs}$ after the device has been switched off. The external bias is that of Fig. 4. Notice the formation of a depletion layer manifested by the "pushing away" of the carrier density away from the gate contact.

distribution obtained at a time $t=6.4 \mathrm{fs}$ after the gate has been switched "off." The early stage of the formation of the depletion layer can clearly be seen (manifested by the "pushing away" of the carrier density from the gate contact). These results, although preliminary, are indicative of the very fast switching times that are believed to characterize the short-channel (submicrometer and ultrasubmicrometer gate) semiconductor devices. Work for the accurate estimation of the switching times of the device along with quantities such as charge and current distributions, charges on the contacts, particle distributions, and energy distributions in the $\Gamma$ and $L$ valleys, is currently under way and results will be reported soon.

\section{CONClusion}

A three-dimensional quantum mechanical simulation method based on the solution of the coupled time-dependent Schrödinger and Poisson equations has been carried out for a planar ultrasubmicrometer gate GaAs MESFET and initial results of the device's transient behavior have been presented. Charge transport in the $\Gamma$ and $L$ conduction band valleys along with intravalley optical and acoustical, and intervalley (equivalent and nonequivalent) phonon scattering has been considered. The effects of phonons are included in an approximation that is consistent with the results of perturbation theory and yields the correct two-point time correlation function in equilibrium. The results obtained indicate that very fast response times characterize such short-channel GaAs devices.

This type of simulation is expected to be particularly useful in the analysis and design of electronic devices with geometric feature sizes comparable to the quantum mechanical wavelength of the carriers, a regime in which the semiclassical approaches are no longer valid and a more fundamental simulation method is needed.

\section{REFERENCES}

[1] D. J. Widiger, I. C. Kizilyalli, K. Hess, and J. J. Coleman, “Twodimensional transient simulation of an idealized high electron mobility transistor," IEEE Trans. Electron Devices, vol. ED-32, pp. 10921102,1985

[2] C. Jacoboni and L. Reggiani, "The Monte Carlo method for the so lution of charge transport in semiconductors with applications to covalent materials," Rev. Mod. Phys., vol. 55, pp. 645-705, 1983.

[3] E. Wigner, "On the quantum correction for thermodynamic equilib rium," Phys. Rev., vol. 40, pp. 749-759, 1932.

[4] G. J. lafrate, H. L. Grubin, and D. K. Ferry, "Utilization of quantum distribution functions for ultra-submicron device transport, " $J$. Phys. suppl. 10, vol. 42, pp. 307-312, 1981.

[5] G. J. Iafrate, "The physics of submicron/ultrasubmicron dimensions," in Gallium Arsenide Technology, D. K. Ferry, Ed. Indianapolis, IN: H. W. Sams \& Co, 1985, ch. 12.

[6] K. K. Thomber and R. P. Feynman, "Velocity acquired by an electron in a finite electric field in a polar crystal," Phys. Rev. B, vol. I, pp. $4099-4114,1970$.

[7] A. O. Caldeira and A. J. Leggett, "Quantum tunneling in a dissipative system," Ann. Phys., vol. 149, pp. 374-456, 1983.

[8] G. D. Mahan, "Quantum transport equation for electric and magnetic fields,'" Phys. Rep., vol. 145, pp. 251-318, 1987.

[9] A. P. Jauho and J. W. Wilkins, "Theory of high-electric-field quantum transport for electron-resonant impurity systems," Phys. Rev. B, vol. 29, pp. 1919-1938, 1984.

[10] G. Bernstein and D. K. Ferry, "Electron beam lithographic fabrication of ultra-submicron gate GaAs MESFETs," Superlattices and Microstructures, vol. 2, pp. 147-150, 1986.

[11] M. A. Littlejohn, J. R. Hauser, and T. H. Glisson, "Velocity-field characteristics of GaAs with $\Gamma_{6}^{\mathrm{c}}-L_{6}^{\mathrm{c}}-X_{6}^{\mathrm{c}}$ conduction band ordering," $J$. Appl. Phys., vol. 48, pp. 4587-4590, 1977

[12] M. C. Yalabik, J. D. Gunton, G. Neofotistos, and K. Diff, “Simulation of charge transport in a GaAs MESFET using the time dependent Schrödinger equation," Superlattices and Microstructures, vol. 3, pp. 463-465, 1987.

[13] W. H. Press, B. P. Flannery, S. A. Teukolsky, and W. T. Vetterling, Numerical Recipes. New York: Cambridge, 1986, p. 386.

[14] R. B. Gerber, R. Kosloff, and M. Berman, "Time-dependent wavepacket calculations of molecular scattering from surfaces," Comp. Phys. Rep., vol. 5, pp. 59-114, 1986. 
M. C. Yalabik, photograph and biography not available at the time of publication.

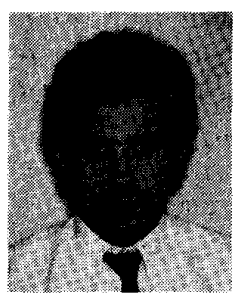

G. Neofotistos received the B.A. degree in physics from the University of Athens, Greece, in 1985. He is working toward the Ph.D. degree in physics at Temple University, Philadelphia, PA. His dissertation is entitled "Quantum Transport in Submicron Semiconductor Electronic Devices." $\mathrm{He}$ is currently conducting research in resonant tunneling in heterojunction structures, device modeling, quantum interference structures, and quantum transport in submicrometer and nanometer-scale GaAs devices.

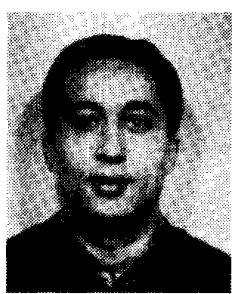

Karim Diff received the B.S. degree in physics from the University of Algiers in 1982, and the M.S. degree in physies from the University of Pennsylvania in 1985 . He is currently working toward the Ph.D. degree in physics at Temple University, Philadelphia, PA. His dissertation will be on quantum charge transport in resonant tunneling heterostructures and he is currently interested in space-charge effects on resonant tunneling, timemodulated barriers, and the energy-loss rates of carriers in quantum wells.

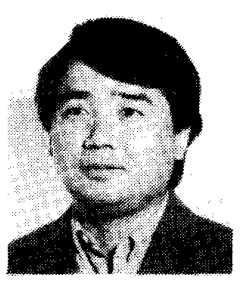

Hong Guo received the B.S. degree in physics from the Sichuan Normal University, and the $\mathrm{Ph} . \mathrm{D}$. degree in physics from the Uniersity of Pittsburgh in 1987 .

His research work has included studies on critical phenomena, finite-size scaling, and renormalization group theories. He is currently interested in quantum transport theory and nonequilibrium statistical physics.

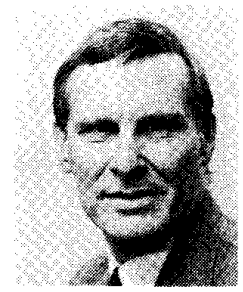

James D. Gunton (M'88) received the B.A. degree in physics from Oxford University (Rhodes Scholar) in 1961 and the Ph.D. degree in physics from Stanford University in 1967.

In 1968, he joined the Department of Physics at Temple University where he was a Professor and Director of the Center for Advanced Computational Science, until July 1988, when he joined Lehigh University as Dean of Arts and Science. His research interests have included renormalization group theories, nonlinear phenomena far from equilibrium, critical dynamics, surface phase transitions, dynamics of metastable and unstable states, finite-size effects, and molecular dynamics simulations of crystal growth. His current research interests are in nonequilibrium statistical physics and quantum transport theory.

Dr. Gunton is a member of the American Physical Society, the New York Academy of Sciences, Sigma Xi, and has served in the Federal Coordinating Council on Science, Engineering and Technology Committee on Supercomputing, and the NSF Advisory Panel on Computational Mathematics. 